Revue internationale P.M.E.

Économie et gestion de la petite et moyenne entreprise

\title{
Le phénomène tontinier au Burkina Faso : étude sur 69 cas
}

\section{Sanov Issoufou}

Volume 5, numéro 3-4, 1992

URI : https://id.erudit.org/iderudit/1008159ar

DOI : https://doi.org/10.7202/1008159ar

Aller au sommaire du numéro

Éditeur(s)

Presses de l’Université du Québec

ISSN

0776-5436 (imprimé)

1918-9699 (numérique)

Découvrir la revue

Citer cet article

Issoufou, S. (1992). Le phénomène tontinier au Burkina Faso : étude sur 69 cas. Revue internationale P.M.E., 5(3-4), 153-170. https://doi.org/10.7202/1008159ar

\section{Résumé de l'article}

Dans la plupart des pays africains, une bonne partie des activités socio-économiques se déroule dans le secteur informel et est financée, par conséquent, par des sources de crédit non officielles (usure, tontine, etc.). La présente étude de cas porte sur la tontine qui est une pratique courante en milieu rural et surtout urbain où le taux de monétarisation est relativement plus important. Ces structures apparaissent aujourd'hui indispensables eu égard à leur contribution dans la mobilisation de l'épargne et la distribution de crédit pour les couches à revenus modestes. Elles suscitent des espoirs réels dans une société où les structures financières modernes répondent encore imparfaitement aux besoins de la grande majorité de la population. 


\title{
Le phénomène tontinier au Burkina Faso : étude sur 69 cas
}

Sanov ISSOUFOU*

CNRST/IRSSH

\begin{abstract}
RÉSUMÉ
Dans la plupart des pays africains, une bonne partie des activités socio-économiques se déroule dans le secteur informel et est financée, par conséquent, par des sources de credit non officielles (usure, tontine, etc.).

La présente étude de cas porte sur la tontine qui est une pratique courante en milieu rural et surtout urbain où le taux de monétarisation est relativement plus important. Ces structures apparaissent aujourd'hui indispensables eu égard à leur contribution dans la mobilisation de l'épargne et la distribution de crédit pour les couches à revenus modestes. Elles suscitent des espoirs réels dans une société où les structures financières modernes répondent encore imparfaitement aux besoins de la grande majorité de la population.
\end{abstract}

\begin{abstract}
In most African countries, a major part of the economic activity takes place within the "informal " sector. It is therefore financed by non-official sources of credit such as pawn shops, tontines.

This case study deals with tontines, a popular instrument in rural areas and even in urban areas where more currency is in circulation.

These financial instruments remain indispensible to collect savings and make loans among the lower middle class. They constitute a real plus in societies whose financial systems still fail to meet adequately the needs of their citizens.
\end{abstract}

* Sanov Issoufou est chercheur au CNRST (Centre national de la recherche scientifique) à Ouagadougou. Il a présenté un mémoire sur le crédit au Burkina Faso en 1985 à l'Université d'Orléans et il poursuit ses recherches dans le cadre des systèmes financiers formel et informel. 


\section{RESUMEN}

En la mayoría de los países africanos, una buena parte de las actividades socioeconómicas se desarrolla en el sector informal, y en consecuencia es financiada mediante fuentes de crédito no oficiales (usura, tontina, etc.).

El presente estudio trata de la tontina como práctica corriente en el medio rural y sobre todo urbano, donde las tasas de monetarización es relativamente más importante.

Estas estructuras parecen hoy en día indispensables debido a su contribución a la mobilización del ahorro y la distribución de créditos a las capas con salarios modestos. Suscitan esperanzas reales en una sociedad donde las estructuras financieras modernas responden todavía de manera imperfecta a las necesidades de la mayoría de la población.

\section{Introduction}

Dans la plupart des pays africains, une bonne partie des activités socio-économiques se déroule dans le secteur informel, financé par conséquent par des sources de crédit non officielles (usure, tontines, etc.). Mais, en dépit de leur importance, ces circuits ont rarement fait l'objet d'une attention particulière, surtout de la part des structures de recherche, de planification et des autorités gouvernementales. Cependant, la connaissance des mécanismes de ces circuits pourrait être utile à bien des égards.

La présente étude portera sur le fonctionnement d'un aspect des circuits informels de crédit: les tontines. Pratique courante en milieu rural, et surtout urbain de nos jours au Burkina Faso, les tontines se créent souvent à partir des réseaux de parenté, de la longue cohabitation dans le voisinage (quartier, service) qui en constituent le support de base (Gerdes, 1975 ; Gracia, 1983).

Ces formes traditionnelles d'épargne et de crédit qui existaient dans la Société a-monétaire, loin de disparaître, ont connu un autre schéma de développement et portent aujourd'hui sur des flux monétaires souvent très importants.

La nature informelle de la tontine exclut a priori une étude par échantillonnage. Il a été préféré une étude de cas très orientée qui, par son caractère intensif, présente un avantage certain en ce qu'elle permet :

- une démarche susceptible de mettre plus en évidence certains mécanismes de fonctionnement touchant au rapport du phénomène avec la société ;

- de dégager des points capables d'apporter un éclairage intéressant à la réflexion théorique (analyse des caractéristiques de la tontine); 
- d'approfondir les investigations compte tenu de la petitesse de l'unité sociale choisie comme champ d'étude ;

- d'étudier de façon précise et suivie l'évolution du sujet afin d'apprécier les différentes transformations subies.

Des enquêtes effectuées principalement dans les deux grands centres urbains de Burkina Faso, Bobo-Dioulasso et Ouagadougou, courant mars 1986, fournissent les données de base à l'étude.

\section{Quelques considérations générales sur la tontine}

\subsection{Définition de la tontine}

Pour un phénomène aussi courant que la tontine, il peut paraître paradoxal de ne pas lui trouver une définition exhaustive. La suivante est assez générale : «Les tontines sont des associations regroupant des membres d'un clan, d'une famille, des voisins ou des particuliers, qui décident de mettre en commun des biens ou des services au bénéfice de tout un chacun, et cela à tour de rôle» (Bouman, 1977 ; Jellicoe, 1968). Il ressort de cette définition deux points essentiels.

Le premier est que les tontines sont largement une inspiration découlant des habitudes communautaires, partie intégrante de la société traditionnelle. La base clanique ou familiale des premières tontines témoigne de l'orientation initiale de ces structures d'entraide qui intervenaient dans tous les aspects de la vie sociale et économique. L'intervention dominante de telles tontines dans les situations d'infortune des populations en ont fait des modes de couvertures indiquées pour les nombreux risques sociaux. Précurseurs des formes d'assurances selon Ottemberg (1955), elles ont pu souvent rendre disponibles des fonds ou des stocks de produits au bénéfice des groupes pour leurs différents besoins sociaux, selon un mode de répartition de la charge qui allégeait le fardeau à chacun (Aubert J., 1983). C'est là un principe qui a pu donner une assez grande quiétude à la vie sociale du milieu fondée sur un esprit solidaire très présent et qui se manifeste surtout à travers des groupes tels que le clan, la famille.

En deuxième point, on entrevoit une ouverture des tontines actuelles à des personnes en dehors des liens (clan, famille, etc.) qui autrefois décidaient de l'appartenance. Cet aspect est très important dès lors qu'il va constituer un facteur du dynamisme des tontines appelées désormais à étendre leurs attributions. C'est ainsi que le motif d'épargne (surtout sous forme monétaire), qui n'était pas dominant dans les premières formes de tontines, sera l'aspect le plus 
en vue dans les nouvelles tontines. La volonté est portée vers une épargne faite en groupe, mais pour des besoins souvent plus personnels (expression de ces besoins et de leur couverture).

\subsection{Motivations en faveur des tontines}

Deux raisons militent en faveur de l'attrait exercé par les tontines :

\section{La raison économique}

La faiblesse des revenus monétaires de la grande majorité de la population rend souvent l'épargne difficile surtout individuellement. La tentation est grande de dépenser rapidement les sommes amassées, avant même qu'elles n'aient atteint le niveau du besoin escompté. Les occasions de dépenses sont nombreuses et l'argent sera soutiré quand bien même il serait dans une tirelire.

La recherche de la formule d'organisation populaire selon Ghilain (1968) et Nicolas (1974), permettant de déjouer d'une part le circuit de l'usure, et, d'autre part, de fournir des moyens de financement peu coûteux, a motivé le recours aux tontines. En effet, elles permettaient l'accumulation des sommes qui représentaient une capacité d'épargne élevée que les tontineurs ne pourraient atteindre en dehors de ce cadre. En imposant aux uns et aux autres une discipline rigoureuse dans l'observation de l'effort d'épargne, au nom de la solidarité mutuelle (Kurtz, 1973), la tontine a suscité ainsi des espoirs. Elle a contribué à stimuler de manière assez significative la volonté d'épargne chez des sujets en général financièrement peu nantis (notamment les groupes défavorisés que sont les femmes et les jeunes). C'est là un aspect progressif et qualitatif de la tontine qui en tant que phénomène social, a dû s'adapter aux nécessités du moment, pour conserver cet atout précieux.

\section{La raison financière}

Au Burkina Faso, une part importante des opérations financières des populations (urbaines comme rurales) s'effectue par le canal des circuits informels d'épargne et de crédit. Ces circuits assurent la mobilisation d'une partie non négligeable des ressources qui est redistribuée le plus souvent directement aux acteurs pour couvrir leurs différents besoins financiers (sociaux, investissements, etc.).

À ce titre, la tontine représente une structure très adaptée qui permet aux membres de réaliser dans les conditions les plus souples le financement de multiples activités économiques. 
La crise économique qui sévit aujourd'hui renforce la conviction des populations dans ces structures qui parviennent malgré tout à régler leurs problèmes financiers.

\section{Trait d'évolution de la tontine}

La force des tontines repose, comme déjà souligné, sur la volonté d'épargne motivée au sein des groupes. Un tel souci était aussi présent dans la société traditionnelle a-monétaire selon $H$. Desroche (1983), cependant que les rapports y étaient beaucoup plus axés sur le social. Cette préoccupation reste encore d'actualité malgré la préférence donnée de plus en plus à l'épargne monétaire. $\mathrm{Ce}$ nouveau changement de cap, en mettant l'accent sur l'aspect financier, n'éclipse pas dans le fond le but suprême de la tontine qui vise ici tout l'homme (social et économique). En effet, la monétisation, puis l'urbanisation, sont apparues comme des facteurs de destruction de la texture de la société traditionnelle. Le besoin de s'affirmer en dehors du cadre habituel assez contraignant a eu pour conséquence la prolifération des tontines, constituées sur de nouveaux rapports. Le profil des nouveaux sociétaires présente le plus souvent des caractéristiques communes (âge et sexe, modicité des ressources, peu d'antagonisme, volonté d'épargne ensemble le plus souvent pour la réalisation d'objectif individuel, etc.).

Ces facteurs ont pu induire des changements dans la forme des tontines (évolution des tontines familiales, classiques ou même ethniques vers des tontines de voisinage, de corporation, d'activité, etc.). Cependant, il reste quant au fond un certain dosage entre le social (constitution de réserves monétaires ou de biens pour réaliser des dépenses sociales telles que : mariage, baptême, frais mortuaires et funérailles, soins médicaux, etc.) et réalisation d'un investissement (constitution d'un fonds de roulement, paiement de la scolarité des enfants, etc.).

\section{Les résultats des enquêtes}

L'enquête a porté sur 80 cas de tontines. Les informations recueillies sur 11 cas étaient sommaires, si bien que ces données n'étaient pas exploitables. Aussi, les 69 cas restants seront en référence de l'étude. 


\subsection{La raison sociale}

Il s'agit là des motifs souvent évoqués pour le regroupement au sein d'une tontine.

- Dans $48 \%$ des tontines, les tontineurs ont déclaré aller dans une tontine dans le but de pouvoir épargner, chose qu'ils n'arrivaient pas toujours à faire en étant seuls. Cette raison émane davantage des hommes $(67 \%$ des cas) que des femmes (seulement $19 \%$ ). Dans les tontines mixtes, ce motif d'épargne était privilégié par $14 \%$ des tontineurs.

- Dans $26 \%$ des tontines, les tontineurs mettent leur participation au compte de l'entraide qui leur apporte, disent-ils, des éléments sécurisants dans la vie sociale. À ce niveau, les femmes dominent largement avec $83 \%$ des cas contre $5 \%$ pour les hommes. Cette raison occupe $12 \%$ des cas dans les tontines réunissant les deux sexes.

- Dans les $26 \%$ restants, les membres avancent un motif purement financier, à savoir miser sur les fonds de la tontine pour lancer ou soutenir une activité (commerce, artisanat et autres services, etc.) ; $57 \%$ de ces tontines sont entre les mains des hommes, $36 \%$ appartiennent aux femmes et $7 \%$ sont mixtes.

\subsection{La nature des liens d'affinité}

La tontine traditionnelle avait basé ses liens sur des considérations ethniques, claniques, tribales ou de parenté étroite. Mais dans le contexte évolutif de la société, ces liens se sont beaucoup effrités surtout en milieu urbain, au profit d'autres formes de polarisation des relations. En effet, les transformations économiques ont entraîné le développement du salariat des activités du tertiaire, et accru les risques sociaux avec les migrations. De ce fait, le besoin de sécurité a pris une grande dimension qui ne pouvait plus être totalement couvert par les mécanismes habituels, d'où l'appel à d'autres formes, soit à titre de compensation, soit pour suppléer un manque total. C'est ainsi que dans les tontines rencontrées, les bases du regroupement semblent avoir été influencées par ces diverses transformations :

- $36 \%$ des tontines sont nées sur le lien du travail (tontines de corporation) ; $88 \%$ de ces dernières regroupent uniquement des hommes contre $12 \%$ de tontines mixtes. Aucune de ces tontines n'enregistre la participation exclusive des femmes.

- $35 \%$ regroupent des personnes intervenant dans une même activité (commerce, artisanat, services, etc.). $49 \%$ de celles-ci sont tenues exclusivement par des femmes, alors que $39 \%$ le sont par des hommes et $13 \%$ par les deux sexes. 
- $23 \%$ des tontines nées dans le voisinage sont des tontines de quartier. Une majorité écrasante de celles-ci ( $88 \%)$ est entre les mains des femmes et $12 \%$ sont des tontines mixtes. On ne note aucune tontine d'hommes à ce niveau.

- $1 \%$ des tontines se rangent sous le couvert familial et $5 \%$ n'ont pas une étiquette précise quant aux liens d'affinité. Le critère de l'affinité apparaît ainsi comme un paramètre beaucoup lié au sexe, au même titre que la raison sociale qu'elle définit.

\subsection{Le sexe}

La séparation entre sexes est un phénomène social auquel n'échappent pas les tontines. Ce clivage était bien accentué dans la société traditionnelle où des barrières et des blocages empêchaient les femmes de participer à toute entreprise aux côtés des hommes avec les mêmes droits et devoirs. Mais avec la monétarisation croissante de l'économie et la participation accrue des femmes au processus de développement (travail salarié, conduite d'activités rémunératrices pour leur émancipation économique), des perspectives de regroupement indépendamment du sexe sont devenues alors possibles. Cependant, des raisons d'affinités et/ou de prudence conduisent encore à l'observation de clivages entre sexes.

C'est ainsi que $51 \%$ des tontines observées seraient tenues par des hommes contre $36 \%$ réservées exclusivement aux femmes et qu'il y aurait $13 \%$ de tontines mixtes.

Les tontines mixtes regroupent en leur sein des membres hommes et femmes. Elles se rencontrent très souvent sur les lieux de service et dans certains milieux professionnels (commerçants, artisans, etc.). Elles ne font aucune discrimination quant au sexe et se fondent uniquement sur la capacité et la volonté de tout membre à participer au but de la tontine.

Ces tontines mixtes traduisent aujourd'hui un effort d'adaptation au contexte nouveau qui voit l'affirmation du rôle économique de la femme et de sa participation égalitaire aux côtés de l'homme.

Le taux élevé des tontines instaurées par les hommes découle du fait qu'un grand nombre des tontines touchées sont sur les lieux de service. La faible représentation des femmes à ce niveau est une situation qui relève de la discrimination qui les frappe sur le marché du travail. Mais dans la vie courante, le degré de participation des femmes aux tontines peut paraître plus élevé que celui des hommes. Cela tient au fait que leur penchant solidaire est plus souvent aiguisé (prédominance des tontines de voisinage) d'une part, et d'autre part, la 
faiblesse de leurs revenus (précarité des situations) les prédispose au choix de ce type de formule pour résoudre dans la dignité leurs nombreux besoins financiers.

\subsection{L'âge}

Dans le même sens que le sexe, le clivage selon l'âge était un aspect visible dans la société traditionnelle où une démarcation systématique pouvait être observée entre aînés et cadets. Mais avec les nouvelles données de la société, cette césure ne semble plus être une barrière en soi. De ce fait, des tontineurs de divers âges peuvent participer à des tontines où l'âge n'est plus un facteur discriminant. Cependant, il convient de remarquer que des regroupements se font toujours selon l'âge des participants, notamment chez certains adolescents ou adultes qui trouvent là un moyen d'affirmer leur autonomie décisionnelle vis-à-vis des anciens.

\subsection{Les modalités de participation}

Outre l'affinité, le sexe et l'âge, qui précisent certaines des modalités de participation à la tontine, le paiement d'un droit d'entrée est un élément de différenciation des tontines.

Ainsi, la grande majorité des tontines rencontrées (86\%) n'applique pas le principe du paiement d'un droit à leurs membres. L'entrée de ces derniers repose sur l'appréciation de leur moralité et de la confiance qu'ils inspirent. Dans ces conditions, on exige que les nouveaux membres soient présentés par un ancien membre ou le président à titre de caution morale. Le paiement d'un droit d'entrée est mentionné par $14 \%$ des tontines. Ce droit très modique, 200 à 500 FCFA (100 FCFA = 2 FF), est perçu et versé dans la caisse où ces fonds restent souvent immobilisés jusqu'à la fin de la tontine. Ceux qui quittent prématurément la tontine perdent le bénéfice du droit versé.

\subsection{Taille des tontines}

La tontine est apparue au début pour les personnes à faibles revenus comme un moyen leur permettant de mettre en commun leur faculté contributive d'entraide et d'épargne. Il va de soi que la tendance ait été à la recherche systématique de groupes élargis. De telles tontines couraient sur une période plus ou moins longue (mois, trimestre, année), au cours de laquelle les sommes restaient bloquées dans une cagnotte. En fin de période, les membres se retrouvaient pour le partage des fonds. 
Ces formes ont laissé la place à celles basées sur la rotation des fonds entre les membres. Le nombre de participants variera dans ces conditions selon la période choisie pour une rotation. Mais, de nos jours, la tontine moderne semble s'orienter vers une structure légère, ce qui donne la préférence aux petits groupes. Ainsi, $65 \%$ des tontines ont moins de 20 membres (30\% entre 2 et 9 membres ; $35 \%$ entre 10 et 19 personnes ; $26 \%$ entre 20 et 50 ; seulement $9 \%$ avaient entre 51 et 150 membres).

\subsection{Les tontines selon la période choisie pour la rotation}

La période choisie pour la rotation revêt une importance réelle dans la mesure où elle influence la taille et même le niveau des cotisations.

Les rotations de durée égale à un mois dominent (43\%), suivies de celles d'une durée comprise entre 2 et 7 jours (35\%). On note une faible fréquence pour les rotations de 8 à 15 jours $(3 \%)$ et celles de plus d'un mois (6\%). Par contre, la période d'une journée semble un peu mieux représentative (13\%). Des paramètres comme la taille, le sexe, l'affinité et le niveau des cotisations, etc., seront mises en relation par la suite pour tenter d'expliquer le choix de la période privilégiée pour la rotation.

\subsection{Les tontines selon le niveau des cotisations}

La participation à une tontine impose au tontineur l'obligation de verser ses cotisations à des échéances fixes ou variables. Cette cotisation, si elle n'est pas supportable par les bourses des membres, peut entraver leur participation de manière durable à la tontine. De ce fait, la fixation du niveau de cotisation relève d'une stratégie qui a pour but de récolter le maximum de fonds au bénéfice des membres d'une part, et d'autre part, d'assurer une longue vie à la tontine. Celle-ci reflétant le plus souvent les conditions et les aspirations de leurs membres, il y a une grande diversité dans les niveaux des cotisations et de leur périodicité.

Dans les tontines rencontrées, les cotisations de 1000 à 5000 FCFA sont dominantes $(42 \%)$. Les petites cotisations de niveau inférieur à $500 \mathrm{~F}$ occupent $32 \%$ des cas. Les cotisations de l'ordre de 5000 et plus représentent $17 \%$ (10\% pour les 5000 à $10000 \mathrm{~F} ; 7 \%$ pour les plus de $10000 \mathrm{~F})$. La tranche de 500 à $1000 \mathrm{~F}$ est peu représentée (seulement $9 \%$ des cas).

\subsection{Les tontines selon le montant des fonds par tournée}

L'avantage de la tontine est qu'elle donne la possibilité aux membres de disposer de sommes d'argent qu'ils n'auraient certainement pas pu amasser s'ils avaient dû 
épargner seuls. Bien que ne mobilisant pas toute la capacité d'épargne des tontineurs, elles constituent cependant des structures par où transitent des sommes non négligeables allouées à chaque bénéficiaire d'un tour dans la tontine.

Selon les résultats des enquêtes, la tranche modale des fonds récoltés à chaque tour est comprise entre 10000 et $20000 \mathrm{~F}(26 \%)$. Les tranches de 20000 à $30000 \mathrm{~F} ; 30000$ à $50000 \mathrm{~F}$ représentent respectivement 20 et $19 \%$. Les niveaux de 50000 à $100000 \mathrm{~F}$ de plus de $100000 \mathrm{~F}$ occupent chacun $10 \%$, et sont assez significatifs comme volume d'épargne et de pouvoir d'achat dans un contexte plutôt difficile.

\subsection{Les tontines selon les modalités d'attribution des tours}

Les fonds reçus dans la caisse de la plupart des tontines sont remis aux différents membres bénéficiaires selon les modalités discutées et arrêtées d'un commun accord, soit au début de la tontine, soit à l'échéance de chaque tour. Ainsi, dans $49 \%$ des tontines, l'attribution des tours se faisait au départ à partir des considérations suivantes : la respectabilité du membre, son honnêteté, le degré de confiance manifesté par le groupe à son égard, sa solvabilité.

Le président de la tontine joue très souvent un rôle capital dans l'attribution des tours par le respect qu'il inspire.

À ce titre, il bénéficie presque toujours du premier tour en signe de la confiance que le groupe manifeste à son égard.

Dans $35 \%$ d'autres cas, le bénéficiaire est tiré à chaque tour. À ce niveau, les tours sont attribués en fonction du caractère pressant des besoins exprimés par chacun des membres. Il peut arriver dans de tels cas que plus d'une personne se prononce pour lever les fonds. Cela nécessite des arbitrages assez serrés pour remettre l'argent du tour sans susciter le moindre mécontentement. Le critère de solvabilité est souvent utilisé pour départager les prétendants. Celui qui présente le plus de garantie peut être privilégié, sauf devant un cas social dûment constaté.

Le système des enchères était pratiqué par $10 \%$ des tontines. Le principe est le suivant :

Les fonds recueillis au titre des cotisations sont mis aux enchères, c'est-àdire mis en vente selon le procédé de marchandage. Seuls les tontineurs n'ayant pas encore bénéficié de leur tour peuvent prendre part aux enchères. C'est le plus offrant, c'est-à-dire la personne qui consent à abandonner dans la caisse la plus forte différence qui enlève les fonds. Exemple : dans une tontine journalière, une douzaine de personnes restaient encore en lice pour les enchères, portant sur des fonds d'un montant de $60000 \mathrm{~F}$. Onze d'entre elles accepteraient seulement de se 
passer de sommes ne dépassant pas $3000 \mathrm{~F}$. La douzième personne a fait monter les enchères jusqu'à $3500 \mathrm{~F}$ et a finalement enlevé les fonds soit : 60000 $3500 \mathrm{~F}=56500 \mathrm{~F}$

La différence constitue le prix à payer pour disposer immédiatement des fonds. Elle n'est pas l'équivalent à proprement parler d'un paiement d'intérêt, mais tout simplement un moyen de dissuasion pour discipliner les membres dans l'attribution des tours. En général, le montant de la différence, élevé au départ de la tontine (beaucoup de prétendants), diminue au fur et à mesure que l'on tend vers la fin du cycle (période pendant laquelle, chaque tontineur a pu disposer de son tour).

Les divers autres cas représentent $6 \%$; il s'agit de situations hybrides qui n'apparaissent pas de façon évidente dans l'une des formes ci-dessus énumérées.

\subsection{Les tontines selon les modalités de remise des fonds}

Les tontines récoltent les contributions de leurs membres. Dans les premières tontines, il semble que ces fonds restaient immobilisés sous la garde du caissier, avant d'être redistribués aux tontineurs selon leurs apports. Mais au regard des problèmes de détournements (de tout ou partie de l'argent de la caisse), les tontines actuelles se présentent plutôt comme des structures par où transitent momentanément des fonds en instance d'être distribués (Bouman, 1976).

C'est ainsi que $86 \%$ des tontines étudiées remettent la totalité des fonds recueillis au bénéficiaire du tour. Aucune somme ne reste disponible dans la caisse, hormis peut-être les cotisations payées comme droit d'entrée (17\% des cas) ou des amendes perçues pour diverses raisons (retards ou absences aux réunions, retards de paiement des cotisations, désordre, etc.).

Dans les $14 \%$ d'autres cas, il y a constitution d'une réserve à chaque tour. Le membre ne reçoit par conséquent qu'une partie des fonds. La réserve représente 2 à $5 \%$ des fonds de la tournée et souvent même plus dans les tontines avec enchères. Les tontineurs en usent souvent pour dépanner les membres qui n'ont pas encore bénéficié de leur tour, pour certains de leurs besoins, ou accessoirement pour payer les cotisations des membres défaillants.

\section{Caractérisation des tontines étudiées}

La présentation ci-dessus s'est bornée à faire apparaître les caractéristiques des tontines repérées. Une brève analyse des résultats permettra d'appréhender certains points de réflexion théorique et pratique sur la tontine à travers le croisement d'un certain nombre de paramètres entre eux. 


\subsection{La raison sociale avec d'autres variables clés}

Il ressort des observations que la nature des affinités conditionne de très près la raison sociale. Aussi, n'est-il pas étonnant que les tontines de voisinage servent davantage le motif entraide (93\% des cas contre $7 \%$ au motif épargne). Pour les tontines de corporation, elles affichent à $100 \%$ la raison épargne, tandis que les tontines issues d'une activité mettent l'accent sur l'aspect financier $(65 \%$ des cas contre $26 \%$ à la raison épargne et $9 \%$ à l'entraide).

En rapport avec la durée de la tournée, il ressort que les tournées hebdomadaires dominent dans le motif entraide : $54 \%$ des cas contre 25 et $21 \%$ respectivement aux motifs épargne et financier. La fréquence de la raison épargne est appréciable dans les tournées mensuelles $(94 \%$ contre $3 \%$ à chacun des deux autres motifs). Quant à la raison financière, elle domine surtout dans les tournées de très courte période (exemple : sur un jour, on compte $67 \%$ des tontines financières).

Le motif de création d'une tontine influe sur la taille dont la dimension tient compte de ce facteur. Ainsi, le motif épargne se rencontre surtout dans les tontines de petite taille, généralement inférieures à 20 membres. Les motifs entraide et financier apparaissent dans les tontines à fortes concentrations $(20$ à 50 personnes) avec une rotation sur des périodes plus courtes (tournée journalière ou hebdomadaire).

La raison sociale appréciée à travers les cotisations laisse apparaître le constat suivant :

Les petites cotisations de l'ordre de 100 à $500 \mathrm{~F}$ sont concentrées sur le motif entraide : $62 \%$ contre 24 et $14 \%$ respectivement aux motifs financier et épargne. Dans les tranches de cotisation de 500 à $1000 \mathrm{~F}$; de 1000 à $5000 \mathrm{~F}$ et de 5000 à $10000 \mathrm{~F}$, le motif épargne domine largement avec 50, 84 et $83 \%$, alors que le motif financier arrive seulement avec 33,10 et $17 \%$. Le motif entraide ne semble pas supporter des cotisations de hauts niveaux.

\subsection{La taille en relation avec d'autres variables}

La relation taille/sexe fait ressortir une préférence assez manifeste des femmes pour les groupes nombreux. C'est ainsi que la taille de 20 à 50 membres ressort plus fréquemment dans les tontines de femmes $(65 \%$ contre $15 \%$ des cas de tontines d'hommes). Cette fréquence atteint $75 \%$ dans les tontines de plus de 50 personnes constituées le plus souvent de femmes. Les hommes qui entretiennent peu de relations de voisinage se retrouvent majoritairement dans des tontines de petite taille (moins de $\mathbf{2 0}$ membres) le plus souvent sur les lieux de travail. 
Le regroupement dans une tontine se fait à partir de l'affinité qui résulte des rapports multidimensionnels existant entre les tontineurs. Dans cet ordre d'idée, la taille des tontineurs de voisinage et celles basées sur l'activité est en majorité de 20 à 50 membres, alors que les tontines de corporation réunissent souvent un nombre inférieur à 20 membres.

La période choisie pour la tournée dépend beaucoup de la taille de la tontine. Le choix judicieux de la période répond au souci de faire en sorte que tous les tontineurs puissent bénéficier des fonds et cela dans un délai qui soit assez raisonnable. L'adéquation du binôme taille-durée de la tournée permet de résoudre ce problème qui n'est pas des moindres. En règle générale, la taille est inversement proportionnelle à la durée de la tournée. C'est ainsi que dans les tournées de durées comprises entre un jour et une semaine, la majeure partie des tontines ont entre 20 et 50 membres et même plus. Par contre, les durées de 8 à 30 jours font ressortir surtout des tailles de moins de 20 membres. On note moins de 10 membres dans les tournées supérieures à 30 jours de durée.

Les cotisations dans les tontines sont modulées en fonction de la taille avec comme objectif, de mettre à la disposition de chaque membre une coquette somme, pour un sacrifice juste supportable. La cotisation, qui repose sur la capacité financière des tontineurs, donne dans sa relation avec la taille une idée de la dimension de l'effort pouvant être demandée à chaque tontineur.

La modicité des cotisations dans les tontines est souvent corrigée par le facteur taille. De ce fait, les petites cotisations de 100 à $500 \mathrm{~F}$ reposent davantage sur des tontines où la taille est élevée (68\% pour les 20 à 50 membres, $18 \%$ pour les plus de 50 membres).

Les cotisations intermédiaires de 500 à $1000 \mathrm{~F}$ se retrouvent dans les différentes tailles, sauf dans les plus de 50 membres. Les cotisations de 1000 à $5000 \mathrm{~F}$ sont très concentrées sur les tailles de moins de 20 membres, alors que dans celles de plus de $5000 \mathrm{~F}$, la taille atteint rarement 10 membres.

Les tontines, en tant que cadre de rapports entre des personnes, ne peuvent échapper à des confrontations qui peuvent dégénérer et porter un coup à ces structures. Bien que fondées sur le principe de la solidarité, des affinités et de la confiance mutuelle, des situations de brouille opposent parfois les tontineurs entre eux. Ces faits souvent rencontrés (cités par environ $30 \%$ des tontines) reposent sur des défaillances partielles ou totales dans le paiement des cotisations, de la non-observation du règlement intérieur ou sur des mésententes entre personnes. La fréquence de tels conflits semble être en rapport avec la taille de la tontine.

Sans présager du caractère absolu et établi de cette relation, il ressort que la fréquence des conflits paraît plus élevée dans les tontines de grande taille. 
Par contre, dans les groupes restreints, la cohésion a pu permettre une certaine discipline. La recherche de petits groupes apparaît comme une stratégie de minimisation des risques de conflits, toute chose qui contribue à assurer une longue vie à la tontine, une fois la confiance établie. C'est un souci qui vaut son pesant d'or quand on sait que la durée de vie moyenne des tontines rencontrées dépasse rarement 3 à 4 ans, cela en référence à la structure et non aux personnes qui la composent (départs et arrivées de nouveaux membres). Le renouvellement incessant des membres pose le problème de la grande instabilité des tontines qui apparaissent bien plus fragiles qu'on ne le pense, en dépit de l'obligation mutuelle qui constitue une pression morale très dissuasive.

\subsection{La cotisation en relation avec d'autres variables}

Le niveau des cotisations en rapport avec le sexe permet d'apprécier les possibilités des tontineurs hommes ou femmes. C'est ainsi qu'on a pu noter une forte concentration de femmes dans les tontines où les cotisations étaient très modestes ; $68 \%$ des tontines de femmes (contre seulement $9 \%$ de tontines d'hommes et $23 \%$ de tontines mixtes) sont dans la tranche de cotisations de 100 à $500 \mathrm{~F}$. Dans les cotisations intermédiaires (500 à 1000 F), il n'y a aucune différence de motivation entre les hommes et les femmes, alors que les tranches supérieures à $1000 \mathrm{~F}$ laissent apparaître la prédominance de tontines d'hommes (surtout pour les cotisations de plus de $10000 \mathrm{~F}$ ). La raison est que les hommes qui participent généralement à la tontine sont pour la plupart des salariés. À l'opposé, les femmes tirent souvent leurs cotisations des activités commerciales, assez peu rémunératrices qu'elles mènent. Ne pouvant compter sur des revenus stables et sûrs à l'instar des salariés, elles sont contraintes de s'engager à des cotisations assez modestes afin de minimiser les risques de non paiement.

La durée choisie pour la tournée corrigée avec la taille permet de fixer en dernier ressort le niveau des cotisations. Ce paramètre revêt ainsi une grande importance, car il permet de mesurer la fréquence de l'effort demandé et de se situer sur les risques liés à cette fréquence. La modicité des revenus monétaires des tontines en général ne leur permet pas de supporter de fortes cotisations, surtout si la périodicité est courte. C'est ainsi qu'on a pu observer que les petites cotisations (100 à $500 \mathrm{~F} ; 500$ à $1000 \mathrm{~F}$ ) sont concentrées sur les plus courtes durées (un jour à une semaine d'intervalle). Ces cotisations se retrouvent dans des tontines de taille élevée. On comprend le risque qu'il y a à allonger la durée qui pourrait pénaliser certains membres, obligés d'attendre très longtemps (plus de 12 mois si la taille est excessive), avant de disposer de leur tour à la tontine. Les cotisations intermédiaires ou élevées courent surtout sur un intervalle mensuel, ce qui semble tout à fait normal. Par contre, un certain nombre de tontines à cotisations de $10000 \mathrm{~F}$ et plus pratiquent des 
durées de rotation supérieures à 30 jours. Ce sont des tontines particulières liées à l'activité réunissant tout au plus 3 à 4 membres, si bien qu'en définitive leur cycle ne dépasse guère 12 mois.

\subsection{Utilisation des fonds reçus de la tontine}

Les tontines mobilisent de l'épargne qui est immédiatement reversée aux membres qui l'utilisent pour résoudre des problèmes sociaux, soutenir une activité économique, acquérir des biens durables et semi-durables. Dans les tontines enquêtées, $38 \%$ des tontineurs ont pu entreprendre une nouvelle activité ou renforcer une activité existante. Il s'agissait le plus souvent d'une activité commerciale (commerce de détail) ou artisanale (artisanat alimentaire, textile, etc.). La plupart de ces initiatives provenaient des femmes.

L'acquisition de biens durables est ressortie dans les déclarations de $15 \%$ des tontineurs. Les hommes semblent avoir une grande préférence pour ce type d'investissement qui touche les aspects suivants : achats de terrains et constructions ; acquisition de moyens de production (outils et équipements). Certains ont même pu entreprendre dans le transport avec l'achat d'un taxi-brousse d'occasion. D'autres ont exploité un verger d'où ils escomptaient tirer une rente substantielle compte tenu du marché souvent rémunérateur des fruits.

L'acquisition de biens semi-durables entre dans la recherche constante d'un certain épanouissement par les ménages : 44,6\% des tontineurs ont affirmé avoir acquis de tels biens avec les fonds reçus pour leur participation à la tontine.

Les hommes valorisaient davantage les moyens de déplacement personnel, le mobilier de maison et les appareils audiovisuels. Les femmes avaient un faible pour l'habillement surtout, l'électroménager et les petits équipements de cuisine.

\subsection{L'intermédiation jouée par la tontine}

Le niveau de bancarisation des populations est resté faible au Burkina Faso où l'attrait des institutions financières formelles n'est pas encore généralisé. Cependant, on remarque que les populations qui ignorent les mécanismes complexes de la finance moderne parviennent à gérer tant bien que mal les tontines qui poussent un peu partout.

La souplesse qui caractérise ce système explique en grande partie sa préférence par les populations (lettrées comme illettrées) qui y font leur apprentissage pour s'imprégner des règles de la finance moderne. 
Au stade actuel, les tontines offrent des facilités aux épargnants (grands et surtout petits) d'accumuler des sommes qui auraient pu être dépensées inutilement et immédiatement. Elles servent ainsi à activer des fonds immobilisés chez les tontiniers. L'habitude des cotisations et la fréquence assez rapprochée accélèrent la circulation de la monnaie et favorisent l'accroissement de la liquidité. Le dynamisme des tontines permet d'envisager ces situations. Par conséquent, leur développement à grande échelle ne peut pas laisser indifférents l'institut d'émission et les institutions financières.

Les tontines arrivent à collecter à moindre coût l'épargne (surtout celle des petits épargnants). Mais elles fonctionnent comme des entités indépendantes les unes des autres, de sorte que leur surface financière se limite à la capacité d'épargne des membres. Par ailleurs, elles ne gardent pas en dépôts les cotisations qui transitent tout juste dans la caisse pour être remises aux bénéficiaires.

Les membres constituent donc des créances non liquides auprès de leur tontine. Cela pose problème quand on sait que les garanties sont souvent inexistantes en cas de défaillance de certains tontineurs. La question qu'on peut se poser alors est la suivante : pourquoi les épargnants constituent-ils leurs créances auprès de ces structures au lieu d'aller vers le système financier moderne où ces créances sont plus liquides et plus sûres?

Cette question laisse apparaître les limites du système tontinier. Les tontines n'ont aucun pouvoir de création monétaire et ne peuvent entreprendre aucune transformation financière à partir de flux transitants. Le crédit est rotatif le plus souvent et correspond à la masse de l'épargne susceptible d'être capitalisée individuellement. Cela amène les uns à cotiser pour les autres en attendant leur tour pour le crédit.

Ces éléments qui constituent des inconvénients sont pourtant acceptés par les tontineurs qui s'y accommodent en se fondant sur l'expérience pour avancer vers des solutions adéquates.

\section{Conclusion}

L'étude a concerné surtout le milieu urbain qui se situe à un stade avancé en ce qui a trait aux tontines monétaires. Le caractère irrégulier du revenu paysan et sa nature saisonnière entravent beaucoup la création des tontines monétaires en milieu rural où les prestations de travail demeurent encore pratiques courantes.

Le champ limité et le nombre réduit de cas étudiés découlent des nombreuses difficultés à pénétrer ce secteur informel où l'information est dissimulée 
et les communications n'y sont pas toujours faciles. En dépit de ces limites, l'étude a pu mettre en exergue certains aspects intéressants de la tontine.

La tontine est un phénomène de masse au Burkina Faso, cependant que cette réalité n'est pas apparue avec une homogénéité certaine dans le temps et dans l'espace en raison des formes variées qu'elle a revêtues. Autrefois basée sur le travail, elle repose aujourd'hui davantage sur la monnaie (milieu urbain surtout) depuis l'introduction des rapports marchands et de la monnaie dans l'économie. Le principe fondé sur les traditions communautaires et l'esprit de solidarité a développé dans un premier temps l'entraide mutuelle pour apporter des soutiens lors d'événements imprévus (décès, mariage, naissance, etc.). Mais de nos jours, des motifs économiques et financiers (épargne et investissement) devenus prépondérants ont par la même occasion rendu nécessaire une adaptation des tontines pour intégrer ces nouveaux aspects. En suscitant le goût à l'épargne surtout au sein des couches à revenus modestes, elles constituent des structures avec lesquelles il faut compter pour la mobilisation des ressources d'épargne. Leur contribution ne saurait être négligée à ce niveau sans préjudice grave. Ce n'est pas tant le volume de cette épargne qui marque l'intérêt pour ces structures, mais surtout le principe qui amène des individus à observer ensemble un penchant naturel comme celui d'épargner, dans le but de s'assurer contre l'imprévu, de réaliser des projets et de diminuer leur propension à dépenser sans but précis. La prolifération des tontines au Burkina Faso nous semble liée à ces raisons, cependant que la difficulté d'accès au crédit bancaire serait à la base de son succès. Puissant mode d'équilibrage, elles suscitent des espoirs certains dans une société où les structures dites modernes répondent encore imparfaitement aux aspirations de la grande majorité de la population.

\section{Bibliographie}

ARDENER, S. (1964), « The cooperative study of rotating associations ", Journal of the Royal Anthropological Institute, vol. 94, p. 201-229.

AUBERT, J.L. (1983), L'assurance-vie et les assurances de personnes, Paris, PUF, Collection Que sais-je ?, $\mathrm{n}^{\circ} 2061$.

Bouman, F.J.A. (1976), « The Djanggi. A traditional form of saving and credit in West Cameroon », Sociologica Ruralis, vol. 16, n 1-2, p. 103-119.

Bouman, F.J.A. (1977), «Indigenous savings and credit in the Third World. A message », Savings and Development, $n^{\circ} 4$, p. 181-219.

CAPLAN, L. (1962), « The multiplication of social ties. The strategy of credit in LDC », Economic Development, vol. 20, ${ }^{\circ} 4$, juillet, p. 691-702. 
Desroche, H. (1983), Solidarité traditionnelle et développement mutualiste, Paris, Actes de l'Université coopérative internationale.

DEZ, J. (1970), « Monnaie et structures traditionnelles », Revue Européenne de Sciences Sociales, Tome 21, p. 176-202.

GeERTZ, C. (1962), «The stating credit associations. A middle rung in development », Economic Development and Cultural Change, vol. 10, $\mathrm{n}^{\circ}$ 3, avril, p. 241-260.

GeRDES, V. (1975), " Precursors of modern social security in indigenous institutions », The Journal of Modern Africa Studies, vol. 13, $\mathrm{n}^{\circ} 2$, p. 209-228.

Ghilain, J. (1968), « À propos de la formation du capital autochtone en Afrique Noire : le likilimba ", Revue de l'Institut de Sociologie, vol. 3, n 2, p. 443-466.

GraCiA, M. (1983), « Auto-organisation des milieux africains et développement », dans Solidarités Traditionnelles et Développement Mutualiste, Université coopérative internationale.

Guy, N. (1974), « La pratique traditionnelle du crédit dans une société sub-saharienne », Culture et Développement, vol. 6, $\mathrm{n}^{\circ} 4$, p. 735-773.

Jellicoe, A.R. (1968), Les associations indigènes d'épargne en Afrique et la mobilisation de l'épargne interne, Addis Abeba, CEA.

KURTZ, D. (1973), « The rotating credit associations. An adaptation to poverty », Human Organisation, vol. $32, \mathrm{n}^{\circ} 1$, printemps, p. 49-58.

Little, K. (1957), « The role of voluntary associations in West African urbanization ", American Anthropologist, vol. 59, p. 51-74.

OTTEMBERG, S. (1955), « Improvement associations among the Afrikpo », Africa, vol. 25, $n^{\circ} 1$. 